\title{
Fatores associados ao bruxismo em crianças de 4 a 6 anos***
}

\author{
Factors associated to bruxism in children from 4 - 6 years
}

\author{
Marcia Simões-Zenari* \\ Mariangela Lopes Bitar**
}

\begin{abstract}
*Fonoaudióloga. Doutora em Saúde Pública pela Faculdade de Saúde Pública da Universidade de São Paulo (USP). Assistente do Curso de Fonoaudiologia do Departamento de Fisioterapia, Fonoaudiologia e Terapia Ocupacional da Faculdade de Medicina (FM) da USP. Endereço para correspondência: R. Cipotânea, 51 São Paulo - SP - CEP 05360-160 (marciasz@usp.br).

**Fonoaudióloga. Doutora em Linguística pela Faculdade de Filosofia, Letras e Ciências Humanas da USP. Docente do Curso de Fonoaudiologia do Departamento de Fisioterapia, Fonoaudiologia e Terapia Ocupacional da FMUSP.

***Trabalho Realizado no Laboratório de Investigação Fonoaudiológica em Promoção da Saúde - Programa Creche do Curso de Fonoaudiologia do Departamento de Fisioterapia, Fonoaudiologia e Terapia Ocupacional da FMUSP.
\end{abstract}

Artigo Original de Pesquisa

Artigo Submetido a Avaliação por Pares

Conflito de Interesse: não

Recebido em 26.02.2010. Revisado em 12.11.2010.

Aceito para Publicação em 19.11.2010.

\begin{abstract}
Background: bruxism has brought losses for the life quality of people. Its implications in the orofacial motricity and speech of children are still not well known. Aim: to investigate bruxism occurrence and associated factors concerning oral habits, orofacial motricity and functions of chewing, breathing and swallowing in children from 4 to 6 years. Method: 141 children from the referred age group who attend three education centers in São Paulo took part in the study. Parents filled in an investigation protocol on bruxism and the children were submitted to an orofacial motricity assessment. The research group was composed by children whose parents indicated habits of teeth clenching or grinding, during sleep or not. For the statistical analysis the Analysis of Variance, the Two-Proportion Equality Test and the Odds Ratio calculation were used, with a significance level of 5\%. Results: a high occurrence of bruxism among the children (55.3\%) was observed. The identified associated factors were: sialorrhea during sleep, pacifier use, habit of lip and fingernails biting, altered cheek tonus and bite, besides the participation of the perioral muscles during liquid swallowing. There was a high occurrence of children from both groups complaining about frequent headaches (76\%) and who slept less hours than what is recommended for their age (35\%). Conclusion: the findings corroborated the relationship among bruxism, oral habits and altered aspects of orofacial motricity in children from the studied age group, reinforcing the necessity of speech therapy actions next to the institutions and families.
\end{abstract}

Key Words: Bruxism, Speech, Child, Health Promotion.

\section{Resumo}

Tema: o bruxismo tem trazido prejuízos para a qualidade de vida das pessoas. Suas implicações para a motricidade orofacial e fala em crianças ainda não estão bem estabelecidas. Objetivo: investigar a ocorrência do bruxismo e fatores associados relativos aos hábitos orais, motricidade orofacial e funções de mastigação, respiração e deglutição em crianças de 4 a 6 anos. Método: participaram 141 crianças da referida faixa etária que frequentam três centros de educação infantil paulistas. Os pais preencheram protocolo de investigação sobre bruxismo e as crianças passaram por avaliação da motricidade orofacial. O grupo pesquisa foi composto pelas crianças cujos pais indicaram qualquer frequência de ranger ou apertamento de dentes, durante o sono ou não. Para análise estatística utilizou-se Análise de Variância, Teste de Igualdade de Duas Proporções e cálculo da Odds Ratio, nível de significância de 5\%. Resultados: observouse elevada ocorrência de bruxismo entre as crianças (55,3\%). Foram fatores associados a esta ocorrência: sialorreia durante o sono, uso de chupeta, hábito de morder lábios e roer unhas, tônus de bochechas e tipo de mordida alterados, além da participação da musculatura perioral durante deglutição de líquidos. Houve alta ocorrência de crianças dos dois grupos com queixa de dor de cabeça frequente (76\%) e que dormem menos do que o recomendado para a idade (35\%). Conclusão: os achados comprovaram relação entre bruxismo, hábitos orais e aspectos alterados da motricidade orofacial das crianças da faixa etária estudada reforçando a necessidade de ações fonoaudiológicas junto às instituições e famílias.

Palavras-Chave: Bruxismo; Fala; Criança; Promoção da Saúde.

Referenciar este material como:

5 Simões-Zenari M, Bitar ML. Fatores associados ao bruxismo em crianças de 4 a 6 anos. Pró-Fono Revista de Atualização Científica. 2010 out-dez;22(4):465-72. 


\section{Introdução}

O bruxismo em crianças tem se tornado uma preocupação crescente nos últimos anos devido ao seu impacto negativo na qualidade de vida e também por ser considerado importante fator de risco para disfunções temporo-mandibulares ${ }^{1-3}$. Pode causar desgaste nos dentes e, em casos mais graves, traumas dentários ${ }^{4}$. Pesquisas recentes têm apontado uma relação entre bruxismo e alterações respiratórias ${ }^{5-7}$. Outras implicações para a motricidade orofacial e fala ainda não estão bem estabelecidas.

Acredita-se que o bruxismo do sono seja mais comum na infância, ainda que não seja incomum em adultos e apresente ocorrência reduzida em idosos ${ }^{3}$. A prevalência desse distúrbio não está precisamente estabelecida, devido às diferenças metodológicas dos estudos ${ }^{4}$, sendo observada em crianças variação de $6 \%$ a $35 \%^{3-4,7-10}$. Estudos longitudinais têm indicado entre 35 e $90 \%$ das crianças com este distúrbio evoluindo com sintomas na idade adulta, segundo revisão de Aloé et al ${ }^{3}$.

Fatores funcionais, estruturais e psicológicos podem estar envolvidos com a presença do bruxismo ${ }^{9,11-12}$. É considerado a atividade parafuncional mais danosa para o sistema estomatognático ${ }^{4}$ e está diretamente relacionado à má qualidade do sono ${ }^{3}$. Dificilmente provoca desgastes dentários importantes em crianças ${ }^{1}$.

O tratamento pode envolver orientações aos pais sobre aspectos comportamentais, acompanhamento odontológico, psicológico e indicação de medicamentos ${ }^{3,13}$. A atuação fonoaudiológica junto a estes casos ainda não é frequente.

Pretende-se, com essa pesquisa, investigar a ocorrência do bruxismo na primeira infância e conhecer os fatores associados relativos aos hábitos orais, aspectos da motricidade orofacial e funções de mastigação, respiração e deglutição. Esses dados possibilitarão maior direcionamento das ações fonoaudiológicas.

\section{Método}

O presente estudo foi aprovado pela Comissão de Ética da instituição de origem (protocolo 085/10). Todos os responsáveis assinaram Termo de Consentimento Livre e Esclarecido, concordando com a participação das crianças na pesquisa e divulgação dos resultados.

Participaram deste estudo de caso-controle 141 crianças que frequentam três centros de educação infantil localizados na região oeste da cidade de São Paulo. Do total de crianças, 71 (50,4\%) são meninos e 70 (49,6\%) são meninas, com idades entre 4:4 anos e $6: 4$ anos, média de 5:4 anos.

Para constituir a amostra foi entregue inicialmente, a todos os pais das crianças entre 4 e 6 anos das referidas instituições, protocolo de investigação de aspectos relacionados à presença de bruxismo. $\mathrm{O}$ protocolo foi desenvolvido para essa pesquisa por não se encontrar na literatura nenhum instrumento validado que pudesse responder às necessidades do estudo. Apresenta questões sobre hábitos orais como ranger dentes, roer unhas, morder lábios, chupar dedo ou chupeta, usar mamadeira, respirar pela boca ou apresentar sialorreia durante o sono. Possibilita que se indique a frequência com que cada aspecto ocorre (se 'sempre', 'às vezes', 'raramente', 'nunca' ou se ‘já ocorreu, mas não ocorre mais'). Além disso, investiga se o sono da criança é tranquilo ou agitado, número médio de horas que dorme, com qual frequência se queixa de dor de cabeça e o que os pais acham de sua personalidade (se é agitada, ansiosa, nervosa, tensa, triste, agressiva, tímida).

Foi solicitado o preenchimento e devolução em, no máximo, uma semana. Dos 287 protocolos enviados houve devolução de 228 (79,4\%).

Todas as crianças cujos pais devolveram os protocolos foram indicadas para avaliação de aspectos da motricidade orofacial. Destas, puderam ser avaliadas 141 (61,8\%), pois a maior parte deixou de frequentar as instituições de um ano para outro.

A avaliação foi realizada nas próprias instituições e consistiu em: verificação de tônus de lábios, língua e bochechas, aparência das tonsilas, da mordida, do palato duro e do freio lingual, postura de repouso de lábios e língua e, ainda, verificação dos padrões de respiração, mastigação e deglutição. Todos esses aspectos foram classificados em adequados ou alterados.

Após esse procedimento foram compostos os grupos pesquisa e controle com todas as 141 crianças que tiveram tanto os protocolos devolvidos quanto a avaliação da motricidade orofacial. Para que a criança fosse incluída no grupo de pesquisa, com queixa de bruxismo (GCB), os pais deveriam ter assinalado alguma ocorrência de ranger de dentes diurno ou noturno no protocolo de investigação. No grupo controle, sem queixa de bruxismo (GSB), entraram as crianças cujos pais indicaram ausência deste comportamento. Este critério foi adotado em conformidade com a literatura sobre o assunto por se tratar de um método simples e objetivo e com a participação dos pais ${ }^{45,7,9,14-16}$.

A análise estatística dos dados utilizou os testes: Análise de Variância (ANOVA) para verificação da homogeneidade dos grupos quanto à idade e sexo para que pudessem ser comparados quanto aos outros aspectos de interesse; Teste de Igualdade de Duas Proporções e cálculo da Odds Ratio (OR) para a comparação dos dois grupos quanto a todos os dados analisados no estudo. Foi considerado nível de significância de 5\%. 


\section{Resultados}

O GCB foi composto por 78 (55,3\%) crianças e o GSB por 63 (44,7\%). Foi testada e comprovada a homogeneidade dos grupos em relação ao sexo $(\mathrm{p}=0,925)$ e à idade $(\mathrm{p}=0,3510)$.

Observou-se elevada ocorrência (55,3\%) de bruxismo relatado pelos pais, sem diferença entre meninos e meninas.

Quando foram comparados os grupos em relação aos hábitos orais (Tabela 1) observaram-se vários aspectos com maior ocorrência no GCB: sialorreia durante o sono ( $\mathrm{p}=0,036)$, uso de chupeta $(\mathrm{p}=0,036)$, morder lábios $(p<0,001)$ e roer unhas $(p=0,028)$. No caso da chupeta, foi encontrada OR de 7,16, ou seja, crianças com esse hábito apresentaram risco sete vezes aumentado de bruxismo. Para as crianças com hábito de morder lábios este risco foi cinco vezes aumentado.
Em ambos os grupos observou-se elevada ocorrência de respiração oral (85\%), sialorreia durante o sono (77\%), morder objetos (51\%) e roer unhas (46\%).

Quanto às características da personalidade não houve diferença. Por outro lado, foi observado elevado número de crianças dos dois grupos consideradas tímidas (66\%), agitadas (50\%), ansiosas (47\%) ou nervosas (31\%).

Em relação aos aspectos miofuncionais orais (Tabela 1), observou-se maior ocorrência de tônus de bochechas ( $p=0,032)$ e tipo de mordida $(p=0,057)$ com alteração no GCB. Além disso, muitas crianças de ambos os grupos apresentaram alteração na postura de repouso de língua (82\%) e de lábios (55\%), no tipo de mordida (53\%), no aspecto das tonsilas (47\%) e do palato duro (43\%) e no tônus de lábio inferior (34\%), de língua (32\%), de lábio superior (28\%) e das bochechas (23\%).

TABELA 1. Comparação dos grupos com e sem bruxismo quanto aos hábitos orais e aspectos miofuncionais orais.

\begin{tabular}{|c|c|c|c|c|c|c|c|}
\hline \multirow{3}{*}{\multicolumn{2}{|c|}{ Parâmetros Avaliados }} & \multicolumn{4}{|c|}{ Grupos } & \multirow{3}{*}{$\begin{array}{l}\text { Valor } \\
\text { de p }\end{array}$} & \multirow{3}{*}{$\begin{array}{l}\text { Odds } \\
\text { Ratio }\end{array}$} \\
\hline & & \multicolumn{2}{|c|}{ Sem Bruxismo } & \multicolumn{2}{|c|}{ Com Bruxis mo } & & \\
\hline & & $\mathrm{n}$ & $\%$ & $\mathrm{n}$ & $\%$ & & \\
\hline \multicolumn{8}{|l|}{ Hábit os Orais } \\
\hline \multirow[t]{2}{*}{ si alorreia durant e o sono } & não & 19 & 30 & 13 & 17 & $0,036 *$ & 2,23 \\
\hline & $\operatorname{sim}$ & 42 & 67 & 64 & 82 & & \\
\hline \multirow[t]{2}{*}{ uso de chupeta } & não & 60 & 95 & 67 & 86 & $0,036^{*}$ & 7,16 \\
\hline & $\operatorname{sim}$ & 1 & 2 & 8 & 10 & & \\
\hline \multirow[t]{2}{*}{ sucção digital } & não & 50 & 80 & 66 & 85 & 0,213 & 0,57 \\
\hline & $\operatorname{sim}$ & 12 & 19 & 9 & 12 & & \\
\hline \multirow[t]{2}{*}{ uso de mamadeira } & não & 50 & 80 & 60 & 77 & 0,744 & 1,17 \\
\hline & $\operatorname{sim}$ & 10 & 16 & 14 & 18 & & \\
\hline \multirow[t]{2}{*}{ morder láb ios } & não & 52 & 83 & 41 & 53 & $<0,001^{*}$ & 4,93 \\
\hline & sim & 9 & 14 & 35 & 45 & & \\
\hline \multirow[t]{2}{*}{ mord er objetos } & não & 36 & 57 & 31 & 40 & $0,040 *$ & 1,97 \\
\hline & sim & 26 & 41 & 44 & 56 & & \\
\hline \multirow[t]{2}{*}{ respiração oral durante o sono } & não & 10 & 16 & 11 & 14 & 0,937 & 1,13 \\
\hline & $\operatorname{sim}$ & 53 & 84 & 66 & 85 & & \\
\hline \multirow[t]{2}{*}{ roer unha } & não & 40 & 64 & 35 & 45 & $0,028 *$ & 2,04 \\
\hline & $\operatorname{sim}$ & 23 & 37 & 41 & 53 & & \\
\hline \multicolumn{8}{|l|}{ Aspectos Miofuncionais Orais } \\
\hline \multirow[t]{2}{*}{ freio lingual } & adequado & 62 & 98 & 76 & 97 & 0,689 & 1,63 \\
\hline & al terado & 1 & 2 & 2 & 3 & & \\
\hline \multirow[t]{2}{*}{ tônus bochechas } & adequado & 54 & 86 & 55 & 71 & $0,032 *$ & 2,51 \\
\hline & al terado & 9 & 14 & 23 & 30 & & \\
\hline \multirow[t]{2}{*}{ tônus lábio inferior } & adequado & 45 & 71 & 48 & 62 & 0,218 & 1,56 \\
\hline & al terado & 18 & 29 & 30 & 38 & & \\
\hline \multirow[t]{2}{*}{ tônus lábio sup erior } & adequado & 45 & 71 & 57 & 73 & 0,828 & 0,92 \\
\hline & al terado & 18 & 29 & 21 & 27 & & \\
\hline \multirow[t]{2}{*}{ tônus língua } & adequado & 46 & 73 & 48 & 62 & 0,151 & 1,74 \\
\hline & al terado & 16 & 26 & 29 & 37 & & \\
\hline \multirow[t]{2}{*}{ postura língua } & adequada & 14 & 22 & 12 & 15 & 0,298 & 1,57 \\
\hline & alterada & 49 & 78 & 66 & 85 & & \\
\hline \multirow[t]{2}{*}{ pos tura lábios } & adequada & 29 & 46 & 34 & 44 & 0,772 & 1,10 \\
\hline & alterada & 34 & 54 & 44 & 56 & & \\
\hline \multirow[t]{2}{*}{ mordida } & adequada & 34 & 54 & 31 & 40 & $0,057 *$ & 1,87 \\
\hline & alterada & 27 & 43 & 46 & 60 & & \\
\hline \multirow[t]{2}{*}{ forma do palato duro } & adequada & 35 & 56 & 45 & 58 & 0,799 & 0,92 \\
\hline & alterada & 28 & 44 & 33 & 42 & & \\
\hline tonsilas & adequadas & 35 & 56 & 36 & 46 & 0,267 & 1,73 \\
\hline & alteradas & 23 & 37 & 41 & 53 & & \\
\hline
\end{tabular}

* estatisticamente significativo 
Quanto ao sono e dor de cabeça (Tabela 2), observou-se que o número insuficiente de horas de sono recomendado para a idade foi associado à presença de bruxismo, com risco aumentado em cerca de cinco vezes. Foi observado elevado número (77\%), nos dois grupos, de crianças com dor de cabeça frequente.

Nas funções de mastigação, deglutição e respiração (Tabela 3), verificou-se que a participação da musculatura perioral durante a deglutição de líquidos ( $\mathrm{p}=0,006$ ) ocorreu mais no GCB.
TABELA 2. Comparação dos grupos com e sem bruxismo quanto à presença de dor de cabeça e aspectos relacionados ao sono.

\begin{tabular}{|c|c|c|c|c|c|c|c|}
\hline \multirow{3}{*}{\multicolumn{2}{|c|}{ Aspectos Investigados }} & \multicolumn{4}{|c|}{ Grupos } & \multirow{3}{*}{$\begin{array}{l}\text { Valor } \\
\text { de } p\end{array}$} & \multirow{3}{*}{$\begin{array}{l}\text { Odds } \\
\text { Ratio }\end{array}$} \\
\hline & & \multicolumn{2}{|c|}{$\begin{array}{c}\text { Sem } \\
\text { Bruxismo }\end{array}$} & \multicolumn{2}{|c|}{$\begin{array}{c}\text { Com } \\
\text { Bruxismo }\end{array}$} & & \\
\hline & & $\mathrm{n}$ & $\%$ & $\mathrm{n}$ & $\%$ & & \\
\hline \multirow{2}{*}{$\begin{array}{l}\text { dor de cab eça } \\
\text { frequente }\end{array}$} & não & 16 & 25 & 15 & 19 & \multirow[t]{2}{*}{0,132} & \multirow[t]{2}{*}{1,53} \\
\hline & $\operatorname{sim}$ & 44 & 70 & 63 & 81 & & \\
\hline \multirow[t]{2}{*}{ acorda à noite } & não & 16 & 25 & 16 & 21 & \multirow[t]{2}{*}{0,491} & \multirow[t]{2}{*}{1,32} \\
\hline & $\operatorname{sim}$ & 47 & 75 & 62 & 80 & & \\
\hline \multirow{2}{*}{$\begin{array}{l}\text { horas de sono } \\
\text { (número) }\end{array}$} & adequado & 51 & 81 & 39 & 50 & \multirow[t]{2}{*}{$<0,001^{*}$} & \multirow[t]{2}{*}{5,10} \\
\hline & alterado & 10 & 16 & 39 & 50 & & \\
\hline
\end{tabular}

TABELA 3. Comparação dos grupos com e sem bruxismo quanto às funções de respiração, mastigação e deglutição.

\begin{tabular}{|c|c|c|c|c|c|c|c|}
\hline \multirow{3}{*}{$\begin{array}{l}\text { Aspect os da Avaliação } \\
\text { das Fun ções }\end{array}$} & & \multicolumn{4}{|c|}{ Grupos } & \multirow{3}{*}{$\begin{array}{l}\text { Valor } \\
\text { de p }\end{array}$} & \multirow{3}{*}{$\begin{array}{l}\text { Odds } \\
\text { Ratio }\end{array}$} \\
\hline & & \multicolumn{2}{|c|}{ Sem Bruxismo } & \multicolumn{2}{|c|}{ Com Bruxismo } & & \\
\hline & & $\mathrm{n}$ & $\%$ & $\mathrm{n}$ & $\%$ & & \\
\hline \multicolumn{8}{|l|}{ Deglutição de Líquidos } \\
\hline movimentação d e cabeça & $\begin{array}{l}\text { ausente } \\
\text { presente }\end{array}$ & $\begin{array}{c}58 \\
2\end{array}$ & $\begin{array}{c}92 \\
3\end{array}$ & $\begin{array}{c}66 \\
8\end{array}$ & $\begin{array}{l}85 \\
10\end{array}$ & 0,177 & 3,52 \\
\hline \multirow[t]{2}{*}{ postura de língua } & adequada & 12 & 19 & 21 & 27 & 0,272 & 0,63 \\
\hline & alt erada & 45 & 71 & 50 & 64 & & \\
\hline \multirow{2}{*}{ parti cipação musculatura perioral } & ausente & 31 & 49 & 21 & 27 & $0,006 *$ & 2,65 \\
\hline & presente & 29 & 46 & 52 & 67 & & \\
\hline \multicolumn{8}{|l|}{ Deglutição de Sólidos } \\
\hline movimentação d e cabeça & $\begin{array}{c}\text { ausente } \\
\text { presente }\end{array}$ & $\begin{array}{c}59 \\
1\end{array}$ & $\begin{array}{c}94 \\
2\end{array}$ & $\begin{array}{c}69 \\
5\end{array}$ & $\begin{array}{c}86 \\
6\end{array}$ & 0,290 & 4,28 \\
\hline \multirow[t]{2}{*}{ postura de língua } & adequada & 13 & 21 & 22 & 28 & 0,301 & 0,63 \\
\hline & alt erada & 46 & 73 & 49 & 63 & & \\
\hline \multirow[t]{2}{*}{ participação musculatura perioral } & ausente & 19 & 30 & 20 & 26 & 0,551 & 1,25 \\
\hline & presente & 41 & 65 & 54 & 69 & & \\
\hline \multirow[t]{2}{*}{ resíduos alimentares } & ausentes & 34 & 54 & 33 & 42 & 0,168 & 1,59 \\
\hline & presentes & 26 & 41 & 40 & 51 & & \\
\hline \multicolumn{8}{|l|}{ Mastigação } \\
\hline \multirow[t]{2}{*}{ lateralização } & presente & 31 & 49 & 32 & 41 & 0,331 & 1,34 \\
\hline & ausente & 29 & 46 & 40 & 51 & & \\
\hline \multirow[t]{2}{*}{ postura de lábios } & adequada & 12 & 19 & 20 & 26 & 0,353 & 0,68 \\
\hline & alt erada & 48 & 76 & 54 & 69 & & \\
\hline \multirow[t]{2}{*}{ movimentação d e mandíbula } & adequada & 40 & 64 & 49 & 63 & 0,935 & 0,94 \\
\hline & alt erada & 20 & 32 & 23 & 30 & & \\
\hline \multirow[t]{2}{*}{ ritmo } & ad equado & 42 & 67 & 59 & 76 & 0,240 & 0,59 \\
\hline & alterado & 18 & 29 & 15 & 19 & & \\
\hline \multicolumn{8}{|l|}{ Respi ração } \\
\hline \multirow[t]{2}{*}{ padrão } & ad equado & 26 & 41 & 33 & 42 & 0,901 & 0,96 \\
\hline & alterado & 37 & 59 & 45 & 58 & & \\
\hline
\end{tabular}

* estatisticamente significativo

\section{Discussão}

Foi encontrada elevada ocorrência de bruxismo em crianças de 4 a 6 anos (55,3\%). Outras pesquisas envolveram faixas etárias mais amplas e diferentes metodologias, dificultando a comparação ${ }^{(3,4,7-10,16)}$. Apenas um estudo apresentou ocorrência semelhante ${ }^{15}$, provavelmente por também considerar como bruxismo o ranger de dentes diurno ou noturno, de ocasional a constante. A escolha por essa classificação considerou que, ainda que $o$ ato de ranger de dentes durante $o$ sono se diferencie em muitos aspectos do apertamento dentário durante a vigília, ambos são frequentes em crianças, com impacto negativo no desenvolvimento ${ }^{3}$.

Optou-se pelo uso de questionário aplicado junto aos pais como sugerido pela literatura ${ }^{4-5,7,7,12,14}$ devido a opção por métodos simples e objetivos para avaliação das crianças, uma vez que estas não têm maturidade para relatar sinais e sintomas de forma precisa ${ }^{9}$. O método de palpação poderia ser empregado, mas seu 
uso ainda é controverso, pois trata-se de exame complexo e difícil de ser analisado pela baixa confiabilidade nas respostas ${ }^{9}$. Eletromiografia não foi considerada como em outros estudos ${ }^{12,17}$ por implicar custos adicionais. Além disso, o foco era a avaliação de crianças na primeira infância e a eletromiografia poderia não ser bem aceita, ainda que não seja um método invasivo. Como a literatura aponta para o hábito de apertar ou ranger dentes como o sinal mais encontrado em estudos com bruxismo ${ }^{18}$ acredita-se que o levantamento deste sinal é suficiente.

A mesma distribuição de bruxismo foi observada entre meninos e meninas, concordando com a literatura ${ }^{6,16}$, o que indica que não há necessidade de ações específicas relacionadas ao gênero.

Quanto aos hábitos orais (Tabela 1), observouse associação entre bruxismo e sialorreia durante o sono, uso de chupeta, morder lábios e roer unhas. Outros estudos também encontraram associação entre bruxismo e morder lábios ${ }^{6}$ e roer unhas ${ }^{6,10}$.

Acredita-se que a associação com sialorreia decorra das relações atualmente discutidas na literatura entre alterações respiratórias e bruxismo, uma vez que a sialorreia pode ser um indicador da respiração oral noturna. Neste estudo não foi encontrada associação entre respiração oral durante o sono e a presença de bruxismo, provavelmente pela elevada frequência de crianças dos dois grupos com padrão respiratório noturno alterado (85\%). A avaliação fonoaudiológica contemplou apenas o padrão respiratório diurno e encontrou 58\% das crianças de ambos os grupos com padrão alterado.

O padrão de respiração precisa ser melhor investigado em estudos futuros, principalmente durante o sono, uma vez que a apneia obstrutiva do sono tem sido considerada como fator de alto risco para distúrbios do sono ${ }^{19}$, entre eles o bruxismo ${ }^{20}$, com melhora significativa deste após adenotonsilectomia ${ }^{5,7}$. Crianças com apneia do sono despertam com frequência, o que aumenta a atividade parafuncional, o que pode levar, entre outros, ao bruxismo, que é associado com sono fragmentado ${ }^{5}$. Há ainda o agravante que crianças com obstrução das vias aéreas tendem a jogar a mandíbula para frente para melhorar a passagem de ar, o que pode estimular receptores das vias aéreas superiores para aumentar tônus das vias aéreas, também levando ao bruxismo ${ }^{5}$.

Do mesmo modo o uso da chupeta, associado ao bruxismo neste estudo, pode sinalizar a presença de respiração oral noturna, uma vez que, ao dormir, a criança tende a soltar a chupeta e a ficar de boca aberta. As crianças que usam chupeta apresentaram risco de bruxismo aumentado em cerca de sete vezes. Outros estudos não encontraram essa relação ${ }^{5-6}$, nem mesmo entre uso de chupeta e alterações temporo-mandibulares ${ }^{10}$. Como em geral muitas crianças usam chupeta nessa idade, fica dificultada a possibilidade de correlacionar este hábito com outros fatores ${ }^{8-9}$, o que não ocorreu neste estudo, onde apenas 6\% do total de crianças usam chupeta.

Como foram elevadas as ocorrências de hábitos orais como morder objetos (50\%), roer unhas (45\%), morder lábios (31\%), usarmamadeira(17\%) e chupar dedo(15\%) pode estar ocorrendo substituição de um hábito por outro, muitas vezes em decorrência de uma retirada abrupta da chupeta. Roer unhas tem sido relacionado com sinais e sintomas de disfunção temporomandibular (DTM) em adolescentes ${ }^{21}$, indicando atenção para este hábito na infância por seus efeitos futuros.

Crianças com hábito de morder os lábios apresentaram risco aumentado em cinco vezes para bruxismo. Assim como em outros estudos, não foi observada associação entre bruxismo e uso de mamadeira ${ }^{5}$ ou sucção digital ${ }^{5-6,16}$.

Também não foi observada associação com características da personalidade das crianças provavelmente por que este dado foi obtido por meio de pergunta direta aos pais, como ocorreu em outro estudo ${ }^{6}$ e não com protocolos mais específicos para avaliar traços da personalidade e comportamento ${ }^{14,22}$. Ainda que esses estudos também sejam considerados limitados, parece haver uma tendência de que as crianças com bruxismo sejam mais tensas e ansiosas ${ }^{16,22}$.

Foi encontrado elevado número (76\%) de crianças com queixa de dor de cabeça frequente (Tabela 2) muito acima dos $23 \%$ observados em outro estudo ${ }^{9}$. Esse fator necessita ser melhor investigado e considerado nas ações futuras junto às famílias e instituições. Dor de cabeça tem sido apontada como uma das queixas de crianças com bruxismo ${ }^{9,21,23}$, associação que não foi encontrada nesta pesquisa provavelmente pela elevada frequência de alteração.

Número de horas de sono aquém do recomendado pela Organização Mundial de Saúde foi associado ao bruxismo (Tabela 2), com risco aumentado em 5 vezes. Crianças de 4 a 6 anos precisam dormir entre 10 e 11 horas por noite, em média, e menos do que isso pode levar à hiperatividade e comportamentos impulsivos, prejudicar a capacidade para aprender e o desempenho acadêmico, pode se relacionar com excesso de peso/obesidade (3 vezes mais risco) e influenciar hormônios reguladores do apetite ${ }^{24}$. Padrão de sono é influenciado pela cultura, nível socioeconômico e estrutura familiar ${ }^{24-25}$. Pais e professores devem conhecer as implicações dos distúrbios do sono para que possam identificar precocemente seus sintomas e frear as consequências, garantindo o bem-estar das 
crianças $^{24-25}$. Problemas de sono menosprezados na infância podem evoluir para problemas psiquiátricos em adultos ${ }^{25}$.

Tônus de bochechas e mordida alterados (Tabela 1) ocorreram com maior frequência nas crianças com bruxismo. Estudo com crianças de 5 a 12 anos encontrou 56\% com mordida aberta e 39\% com mordida cruzada, mas sem associação com bruxismo ou DTM ${ }^{9}$. Outro estudo encontrou redução do bruxismo após adenotonsilectomia, sem que houvesse mudança na oclusão ${ }^{5}$. Esses dados indicam que essas alterações podem estar associadas, sem que haja uma relação de causalidade entre elas.

A participação da musculatura perioral durante a deglutição de líquidos (Tabela 3) ocorreu mais nas crianças com bruxismo o que pode indicar contração excessiva dessa musculatura quando desnecessário, talvez pelo apertamento dentário existente. Dor ao abrir a boca ou mastigar foi mais presente em crianças com bruxismo em estudo realizado com crianças entre 8 e 12 anos $^{22}$. Hipertrofia dos músculos mastigatórios tem sido observada em casos de bruxismo ${ }^{12,26}$ e o padrão mastigatório unilateral pode estar relacionado com DTM ${ }^{2}$. Estudo com crianças entre 2 e 13 anos com obstrução nasal não observou diferença quanto à presença de dor para mastigar naquelas com bruxismo, mas consideram a possibilidade dos episódios de bruxismo serem ocasionais e suaves em crianças e que os sintomas podem se manifestar mais tarde se o bruxismo persistir como um fator agressivo contra o sistema estomatognático ${ }^{6}$.

Os resultados indicam necessidade de ações junto aos pais e instituições. A ocorrência do bruxismo entre as crianças deve ser valorizada e intervenções eficazes devem ser desenvolvidas. Observar sintomas nas crianças pode prevenir disfunções futuras como dor e desconforto ${ }^{10}$.

Tanto a ocorrência de bruxismo quanto o hábito de fumar devem ser levantados entre as famílias. Traço de hereditariedade é um indicador importante ${ }^{3,16}$ e fumantes têm risco duas vezes aumentado para bruxismo ${ }^{3}$.

Atualmente o tratamento do bruxismo tende a ser multiprofissional ${ }^{12}$ envolvendo aspectos comportamentais - controle da ansiedade e melhoria dos hábitos de sono, odontológicos - principalmente ajustes oclusais e uso de placas, psicológicos e indicação de medicamentos - relaxantes musculares, antidepressivos e outros ${ }^{3,13}$. A avaliação fonoaudiológica se mostrou muito importante pela possibilidade de se verificar o impacto do bruxismo na motricidade orofacial, funções alimentares e respiração e por possibilitar o delineamento de ações nessa área.

\section{Conclusão}

Foi encontrada elevada ocorrência de bruxismo e importantes aspectos apresentaram-se associados a este distúrbio, fatores que justificam o desenvolvimento de ações fonoaudiológicas junto às crianças, famílias e instituições. 


\section{Referências Bibliográficas}

1. Gusson DGD. Bruxismo em crianças. J. Bras. Odontopediatr. Odontol. Bebe. 1998; 1(2):75-97.

2. Merighi LBM, Silva MMA, Ferreira AT, Genaro KF, Berretin-Felix G. Ocorrência de disfunção temporomandibular (DTM) e sua relação com hábitos orais deletérios em crianças do município de Monte Negro - RO. Rev. Cefac. 2007; 9(4):497-503.

3. Aloé F, Gonçalves LR, Azevedo A, Barbosa RC. Bruxismo durante o sono. Rev. Neurociências. 2003;11(1):4-17.

4. Koyano K, Tsukiyama Y, Ichiki R, Kuwata T. Assessment of bruxism in the clinic. J Oral Rehabil. 2008;35:495-508.

5. DiFrancesco RC, Junqueira PAS, Trezza PM, Faria MEJ, Frizzarini R, Zerati FE. Improvement of bruxism after T \& A surgery. Int J Pediatr Otorhinolaryngol. 2004;68: 441-5.

6. Grechi TH, Trawitzki LVV, Felicio CM, Valera FCP, Anselmo-Lima WT. Bruxism in children with nasal obstruction. Int J Pediatr Otorhinolaryngol. 2008;72:391-6.

7. Eftekharian A, Raad N, Gholami-Ghasri N. Bruxism and adenotonsillectomy. Int J Pediatr Otorhinolaryngol. 2008;72:509-11.

8. Galvão ACUR, Menezes SFL, Nemr K. Correlação de hábitos orais deletérios entre crianças de 4 a 6 anos de escola pública e escola particular da cidade de Manaus - AM. Rev CEFAC. 2006;8(3):328-36.

9. Santos ECA, Bertoz FA, Pignatta LMB, Arantes FM. Avaliação clínica de sinais e sintomas da disfunção temporomandibular em crianças. R Dental Press Ortodon Ortop Facial. 2006;11(2):29-34.

10. Tosato JP, Biasotto-Gonzalez DA. Symptomatology of the temporomandibular dysfunction related to parafunctional habits in children. Braz J Oral Sci. 2005;4 (14):787-90

11. Seraidarian P, Seraidarian PI, Cavalcanti BN, Marchini L, Neves ACC. Urinary levels of catecholamines among individuals with and without sleep bruxism. Sleep breath 2009;13:85-8.

12. Barbosa TS, Miyakoda LS, Pocztaruk RL, Rocha CP, Gavião MBD. Temporomandibular disorders and bruxism in childhood and adolescence: review of the literature. Int J Pediatr Otorhinolaryngol. 2008;72:299-314.

13. Lobbezoo F, Van der Zaag J, Van Selms MKA, Hamburguer HL. Principles for the management of bruxism. J Oral Rehabil. 2008;35:509-23.
14. Ghanizadeh A. ADHD, bruxismo and psychiatric disorders: does bruxism increase the chance of a comorbid psychiatric disorder in children with ADHD and their parents? Sleep breath. 2008;12:375-80.

15. Moore M, Allison D, Rosen CL. A review of pediatric nonrespiratory sleep disorders. Chest. 2006;130(4):1252-62.

16. Cheifetz AT, Osganian SK, Allred EN, Needleman HL. Prevalence of bruxism and associated correlates in children as reported by parents. J Dent Child. 2005;72(2):67-73.

17. Lavigne GJ, Khoury S, Abe S, Yamaguchi T, Raphael K. Bruxism physiology and pathology: an overview for clinicians. J Oral Rehabil. 2008;35:476-94.

18. Martins RJ, Garcia AR, Garbin CAS, Sundefeld MLMM. Associação entre classe econômica e estresse na ocorrência da disfunção temporomandibular. Rev. Bras. Epidemiol. 2007;10(2):215-22.

19. Carneiro LEP, Neto GCR, Camera MG. Efeito da adenotonsilectomia sobre a qualidade de vida de crianças com hiperplasia adenotonsilar. Arq. Int. Otorrinolaringol. 2009;13 (3):270-6.

20. Ohayon MM, Li KK, Guilleminault C. Risk factors for sleep bruxism in the general population. Chest. 2001;119(1):53-61.

21. Feteih RM. Signs and symptons of temporomandibular disorders and oral parafunctions in urban Saudi arabian adolescents: a research report. Head \& Face Medicine. 2006;2:25

22. Restrepo CC, Vásquez LM, Alvarez M, Valencia I. Personality traits and temporomandibular disorders in a group of children with bruxing behaviour. J Oral Rehabil. 2008;35:585-93.

23. Gorayeb MAM, Gorayeb R. Cefaléia associada a indicadores de transtornos de ansiedade em uma amostra de escolares de Ribeirão Preto, SP. Arq Neuropsiquiatr. 2002,60(3-B):764-8.

24. Touchette E, Petit D, Tremblay RE, Montplaisir JY. Risk factors and consequences of early childhood dyssomnias: new perspectives. Sleep Medicine Reviews. 2009;13:355-61.

25. Klein JM, Gonçalves A. Problemas de sono-vigilia em crianças: um estudo da prevalência. Psico-USF. 2008;13(1):51-8.

26. Díaz-Serrano KV, Silva CBA, Albuquerque S, Saraiva MCP, Nelson-Filho P. Is there na association between bruxism and intestinal parasitic infestation in children? J Dent Child. 2008;75(3):276-9. 